\title{
Treadmill exercise in obese maternal rats during pregnancy improves short-term memory through neurogenesis in the hippocampus of rat pups
}

\author{
Eun-Sang Ji',2, You-Mi Kim³ ${ }^{3}$ Young Jun Ko ${ }^{4}$, Seung-Soo Baek ${ }^{1, *}$ \\ 'Department of Sport \& Health Care, College of Art \& Culture, Sangmyung University, Seoul, Korea \\ ${ }^{2}$ Department of Physiology, College of Medicine, Kyung Hee University, Seoul, Korea \\ ${ }^{3}$ Sports Science Research Institution, Korea National Sport University, Seoul, Korea \\ ${ }^{4}$ Major in Sport Service Practice, College of Welfare Convergence, Kangnam University, Yongin, Korea
}

Maternal obesity is known to increase the likelihood of offspring becoming obese, high blood pressure, and other metabolic disorders. After inducing obesity, the effect of treadmill exercise in maternal rats during pregnancy on short-term memory was investigated in relation to neurogenesis in rat pups. Short-term memory was declined in rat pups born to obese maternal rats, and treadmill running during pregnancy alleviated short-term memory impairment in rat pups born to obese maternal rats. The number of doublecortin (DCX)-positive and 5-bromo-2'-deoxyuridine (BrdU)-positive cells in the hippocampal dentate gyrus was decreased in rat pups born to obese maternal rats. Treadmill running during pregnancy increased the number of DCX-positive and
BrdU-positive cells in rat pups born to obese maternal rats. Expression of brain-derived neurotrophic factor (BDNF) and tyrosine kinase B (TrkB) in the hippocampus was decreased in the rat pups born to obese maternal rats. Treadmill running during pregnancy increased the expressions of BDNF and TrkB in rat pups born to obese maternal rats. Enhancing effect of short-term memory by treadmill exercise may be due to increased neurogenesis through activation of the BDNF-TrkB signaling pathway by treadmill exercise.

Keywords: Maternal obesity, Pregnancy, Treadmill exercise, Hippocampus, Neurogenesis, Rat pups

\section{INTRODUCTION}

Maternal health during pregnancy refers to the health of the fetus, and maternal obesity is known to have a direct effect on the induction of diabetes in children (Tozuka et al., 2010). Maternal obesity is known to increase the likelihood of offspring becoming obese, high blood pressure, and other metabolic disorders (Chang et al., 2008; Shankar et al., 2008). Reduced brain-derived neurotrophic factor (BDNF) expression, impaired spatial cognitive function, and suppressed neurogenesis in the hippocampus have been observed in pups born to obese maternal rats (Tozuka et al., 2010). The hippocampus is a component of the brain limbic system and the hippocampus incorporates all information such as short-term memory, long-term memory, spatial learning memory, and spatial working memory (Snyder et al., 2005).

Various neuronal markers appear on developing neurons. Immunohistochemistry for 5-bromo-2'-deoxyuridine (BrdU) is used for quantification of new cell generation (Park et al., 2019), and the neuronal nuclear $(\mathrm{NeuN})$ antigen is considered as a useful marker for neuronal maturation (Park et al., 2019). Doublecortin (DCX) is a microtubule-related protein and specifically expressed during cell production in the central nervous system (Couillard-Despres et al., 2001). DCX expression refers a marker of neuronal precursor cells and indicates the migration and development of neurons (Park et al., 2019).

BDNF regulates neuronal development, differentiation, and survival through the receptor tyrosine kinase B (TrkB), and BDNF is secreted by activity-dependent modulators (Massa et al., 2010).
${ }^{*}$ Corresponding author: Seung-Soo Baek (iD https://orcid.org/0000-0002-1340-2098 Department of Sport \& Health Care, College of Art \& Culture, Sangmyung University, 20 Hongjimun 2-gil, Jongno-gu, Seoul 03016, Korea

E-mail: ssoop@smu.ac.kr

Received: August 20, 2020 / Accepted: September 25, 2020
This is an Open Access article distributed under the terms of the Creative Commons Attribution Non-Commercial License (https://creativecommons.org/licenses/by-nc/4.0/) which permits unrestricted non-commercial use, distribution, and reproduction in any medium, provided the original work is properly cited. 
Learning and memory skills in rat pups were increased with exercise of maternal rats during pregnancy (Akhavan et al., 2008), and low-intensity exercise of maternal rats during pregnancy was associate with improved memory function of pups born to maternal rats with posttraumatic stress disorder (Seo et al., 2013).

There are insufficient scientific evidences for the effect of exercise on short-term memory in children during pregnancy in obese mothers. In this study, step-down avoidance task was performed to assess the impact of treadmill exercise in obese maternal rats during pregnancy on the short-term memory of rat pups. The expression of BrdU and DCX was detected by immunohistochemistry and the expression of BDNF and TrkB, was measured by western blot analysis.

\section{MATERIALS AND METHODS}

\section{Animals}

Approval number was achieved from the Kyung Hee University Institutional Animal Care and Use Committee in Seoul, Korea (KHUASP [SE]-17-099). The experimental animals used in this study were 4-week-old Sprague-Dawley male rats $(n=8)$ and female rats $(\mathrm{n}=16)$. In order to induce obesity, female rats were supplied with the high-fat diet (60\% fat) purchased from Central Laboratory Animals (Seoul, Korea). Female rats were classified into the normal diet group $(n=8)$ and the high-fat diet group $(n=8)$ for a total of 7 weeks, including gestation period and lactation period. Female rats were bred individually $48 \mathrm{hr}$ after mating with male rats. Female rats with confirmed pregnancy were divided into normal diet group, normal diet and treadmill exercise group, high-fat diet group, high-fat diet and treadmill exercise group. The born rat pups were divided into control group, treadmill exercise group, high-fat diet group, high-fat diet and treadmill exercise group ( $\mathrm{n}=10)$, according to the classification status of maternal rats. All experiments were conducted in compliance with the recommendations of the National Institutes of Health and the Korean Academy of Medical Sciences.

\section{Treadmill exercise protocol and BrdU injection}

The running speed of treadmill during the pregnancy was $3 \mathrm{~m} /$ $\min$ for the first $5 \mathrm{~min}, 5 \mathrm{~m} / \mathrm{min}$ for the next $5 \mathrm{~min}, 8 \mathrm{~m} / \mathrm{min}$ for the last $20 \mathrm{~min}, 1$ time for $30 \mathrm{~min}, 5$ times a week, and for 4 weeks. BrdU (Sigma Chemical Co., St. Louis, MO, USA) was injected intraperitoneally with $50 \mathrm{mg} / \mathrm{kg}$ once a day for 5 days from the 15 th day of pregnancy $60 \mathrm{~min}$ before the treadmill exercise.

\section{Step-down avoidance task}

In the same manner, as described below (Ko et al., 2019), the latency in the step-down avoidance task was measured to assess short-term memory. The rat pups were trained with a step-down avoidance task after last treadmill exercise. The rat pups were placed $7 \times 25 \times 2.5-\mathrm{cm}$ platform and the platform faced a $42 \times 25-\mathrm{cm}$ grid of parallel $0.1-\mathrm{cm}$ caliber stainless-steel bars spaced $1 \mathrm{~cm}$ apart. In training session, the animals were subjected to a $0.5-\mathrm{mA}$ scramble foot shock for 2 sec as soon as they descended. One hour after training, the latency (in seconds) of each animal was measured. We defined the latency as the time interval between when the rat descends and puts all four feet on the grid, and a latency of more than $300 \mathrm{sec}$ was calculated as $300 \mathrm{sec}$.

\section{Tissue preparation}

Immediately after the Morris water maze task, the rat pups were anesthetized with intraperitoneal injection of Zoletil $50(10 \mathrm{mg} / \mathrm{kg}$, Vibac Laboratories, Carros, France). The rat pups were cardiac perfused with 50-mM phosphate-buffered saline (PBS) and then fixed by $4 \%$ paraformaldehyde dissolved in $100-\mathrm{mM}$ phosphate buffer ( $\mathrm{PB} ; \mathrm{pH}, 7.4)$. After the brains were removed, the brains were postfixed overnight using the same fixative and transferred to a $30 \%$ sucrose solution to prevent freezing. The $40-\mu \mathrm{m}$ thick sagittal sections were created using a freezing microtome (Leica, Nussloch, Germany).

\section{Immunohistochemistry for DCX}

In the same manner, as described below, immunohistochemistry for DCX was performed (Park et al., 2019). The sections were treated with PBS for 10 min and then treated again with $1 \% \mathrm{H}_{2} \mathrm{O}_{2}$ for $20 \mathrm{~min}$. The sections were treated with goat anti-DCX antibody (1:1,000; On-cogene Research Product, Cambridge, UK) for $2 \mathrm{hr}$. The sections were treated with the biotinylated goat secondary antibody (1:500; Vector Laboratories, Burlingame, CA, USA) for another $1 \mathrm{hr}$, washed, and treated with $\mathrm{ABC}$ complex (1:100; Vector Laboratories). Using the $0.03 \%$ diaminobenzidine, labeling was visualized and the sections were mounted onto gelatin-coated slides. After air drying the slides at room temperature overnight, the coverslips were mounted using Permount (Thermo Fisher Scientific Inc., Waltham, MA, USA).

\section{Immunohistochemistry for BrdU}

In the same manner, as described below, immunohistochemistry for BrdU was performed (Park et al., 2019). The sections were first permeabilized by treating with $0.5 \%$ Triton X-100 in PBS 
for $20 \mathrm{~min}$, then treated with $50 \%$ formamide- $2 \mathrm{X}$ standard saline citrate at $65^{\circ} \mathrm{C}$ for $2 \mathrm{hr}$, denatured in $2 \mathrm{~N} \mathrm{HCl}$ at $37^{\circ} \mathrm{C}$ for 30 min, and washed twice in 100-mM sodium borate ( $\mathrm{pH}, 8.5)$. The sections were treated with BrdU-specific mouse monoclonal antibody (1:600; Roche, Mannheim, Germany) at $4^{\circ} \mathrm{C}$ overnight. The sections were treated with biotinylated mouse secondary antibody (1:200; Vector Laboratories) for $1 \mathrm{hr}$ and treated with ABC complex (1:100; Vector Laboratories) for $1 \mathrm{hr}$. To visualized, the sections were treated for $5 \mathrm{~min}$ in $50-\mathrm{mM}$ Tris- $\mathrm{HCl}(\mathrm{pH}, 7.6)$ containing $0.03 \%$ diaminobenzidine, $0.03 \% \mathrm{H}_{2} \mathrm{O}_{2}$ and $40-\mathrm{mg} / \mathrm{mL}$ nickel chloride.

To differentiate neurons, mouse antineuronal nuclei antibody (1:1,000; Chemicon International, Temecula, CA, USA) was used in the same sections after BrdU labeling. The sections were treated with a biotinylated anti-mouse secondary antibody for $1 \mathrm{hr}$. For staining, the sections were treated in a reaction mixture consisting of $0.03 \%$ diaminobenzidine and $0.03 \% \mathrm{H}_{2} \mathrm{O}_{2}$ for $5 \mathrm{~min}$. The sections were mounted onto gelatin-coated slides, air-dried overnight at room temperature, and coverslips were mounted using Permount (Thermo Fisher Scientific Inc.).

\section{Western blot analysis}

In the same manner, as described below, western blotting for the determination of BDNF and TrkB was conducted (Park et al., 2019). Hippocampal tissues were homogenized on ice and lysed in a lysis buffer consisting of 1-mM phenylmethylsulfonyl fluoride, $150-\mathrm{mM} \mathrm{NaCl}, 0.1 \%$ sodium dodecyl sulfate (SDS), $0.5 \%$ deoxycholic acid, $1 \%$ Nonidet P40, 100-mg/mL leupeptin, 50-mM Tris- $\mathrm{HCl}(\mathrm{pH}, 7.5)$. Colorimetric protein assay kit (Bio-Rad, Hercules, CA, USA) was used to measure protein content. Total protein $30 \mu \mathrm{g}$ was separated on SDS-poly-acrylamide gels and transferred to a nitrocellulose membrane, the membrane was blocked with dehydrated milk and then treated with primary antibodies. Mouse anti- $\beta$ actin (1:3,000; Santa Cruz Biotechnology, CA, USA), rabbit anti-BDNF (1:1,000; Santa Cruz Biotechnology), and rabbit anti-TrkB (1:1,000; Santa Cruz Biotechnology) were used as the primary antibody.

After washing, appropriate horseradish peroxidase-conjugated secondary antibodies were used and incubation was performed at room temperature. Enhanced chemiluminescence detection system (Santa Cruz Biotechnology) was used to measure the expression of bands, and bands were quantified using an Image-Pro Plus computer-assisted image analysis system (Media Cyberbetics Inc., Silver Spring, MD, USA).

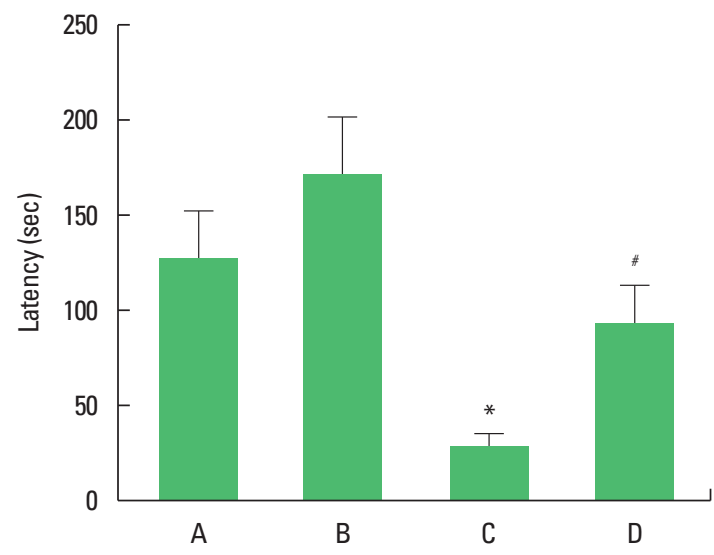

Fig. 1. Short-term memory of the rat pups. A, control group; B, treadmill exercise group; $C$, high-fat diet group; $D$, high-fat diet and treadmill exercise group. ${ }^{*} P<0.05$ compared with the control group. ${ }^{\sharp} P<0.05$ compared with the highfat diet group. Data represents the mean \pm standard error of the mean.

\section{Data analysis}

IBM SPSS Statistics ver. 21.0 (IBM Co., Armonk, NY, USA) was used to analyze the data, and one-way analysis of variance using the Duncan post hoc test was used for comparisons between groups. The results were presented as the mean \pm standard error of the mean, and $P<0.05$ was shown to be statistically significant.

\section{RESULTS}

\section{Short-term memory}

In order to investigate the effect of treadmill exercise during pregnancy of obese maternal rats on the short-term memory in rat pups, a step-down avoidance task was performed (Fig. 1). In the high-fat diet group, the latency was significantly reduced compared with the control group $(P<0.05)$. Treadmill running was found to significantly increase the latency in the high-fat diet group $(P<0.05)$.

\section{Number of DCX-positive cells}

In order to investigate the effect of treadmill exercise during pregnancy of obese maternal rats on the neuronal production in hippocampal dentate gyrus of rat pups, the number of DCX-positive cells was observed (Fig. 2). The number of DCX-positive cells was significantly reduced compared with the control group $(P<$ $0.05)$. Treadmill running was found to significantly increase the number of DCX-positive cells in the high-fat diet group $(P<0.05)$.

\section{Number of BrdU-positive cells}

In order to investigate the effect of treadmill exercise during 

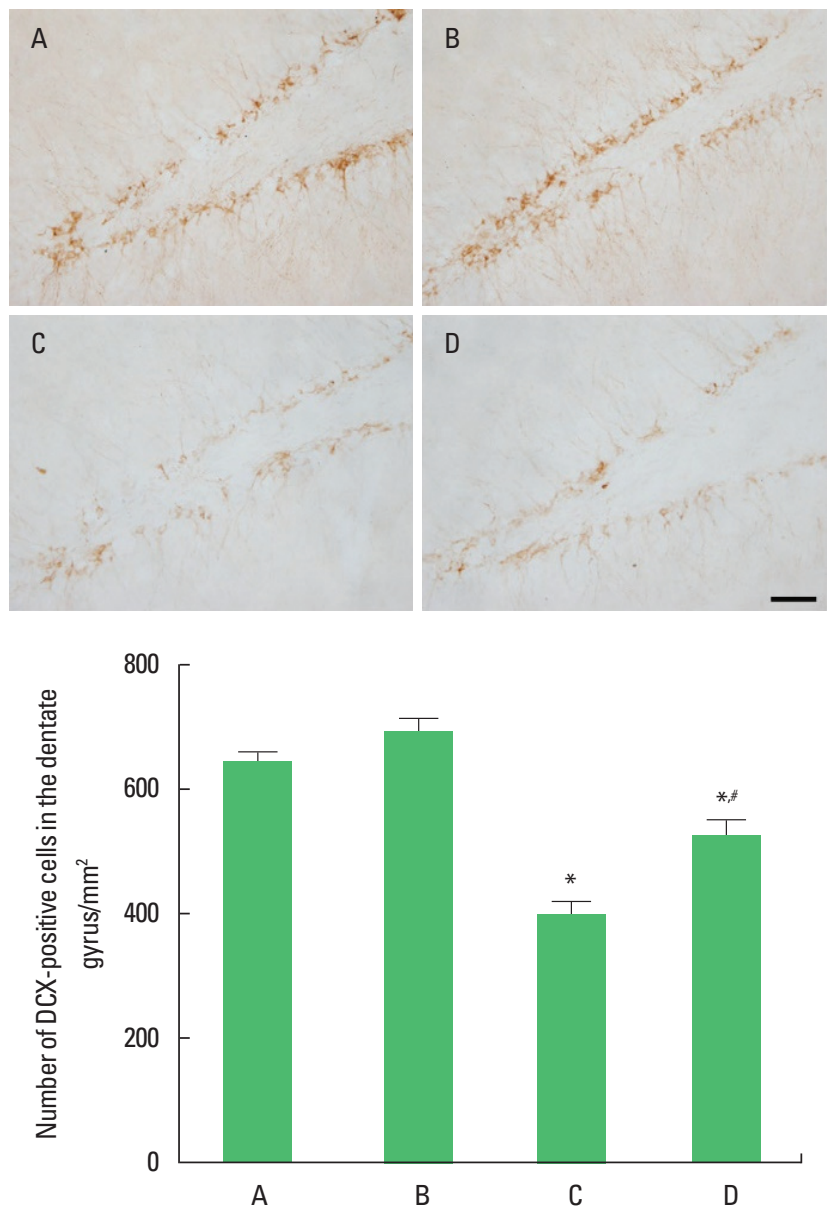

Fig. 2. Doublecortin (DCX) expression in the hippocampal dentate gyrus of rat pups. Upper panel: photomicrographs of DCX-positive cells. The scale bar represents $50 \mu \mathrm{m}$. Lower panel: number of DCX-positive cells in each group. $A$, control group; $B$, treadmill exercise group; $C$, high-fat diet group; $D$, high-fat diet and treadmill exercise group. ${ }^{*} P<0.05$ compared with the control group. ${ }^{\#} P<0.05$ compared with the high-fat diet group. Data represents the mean \pm standard error of the mean.

pregnancy of obese maternal rats on the cell proliferation in hippocampal dentate gyrus of rat pups, the number of BrdU-positive cells was observed (Fig. 3). The number of BrdU-positive cells was significantly reduced compared with the control group $(P<$ $0.05)$. Treadmill running was found to significantly increase the number of BrdU-positive cells in the high-fat diet group $(P<0.05)$.

\section{BDNF and TrkB expression}

In order to investigate the effect of treadmill exercise during pregnancy of obese maternal rats on the expression of BDNF and TrkB in the hippocampus of rat pups, the expression of BDNF and TrkB was observed (Fig. 4). The expression of BDNF and TrkB was significantly reduced in the high-fat diet group compared
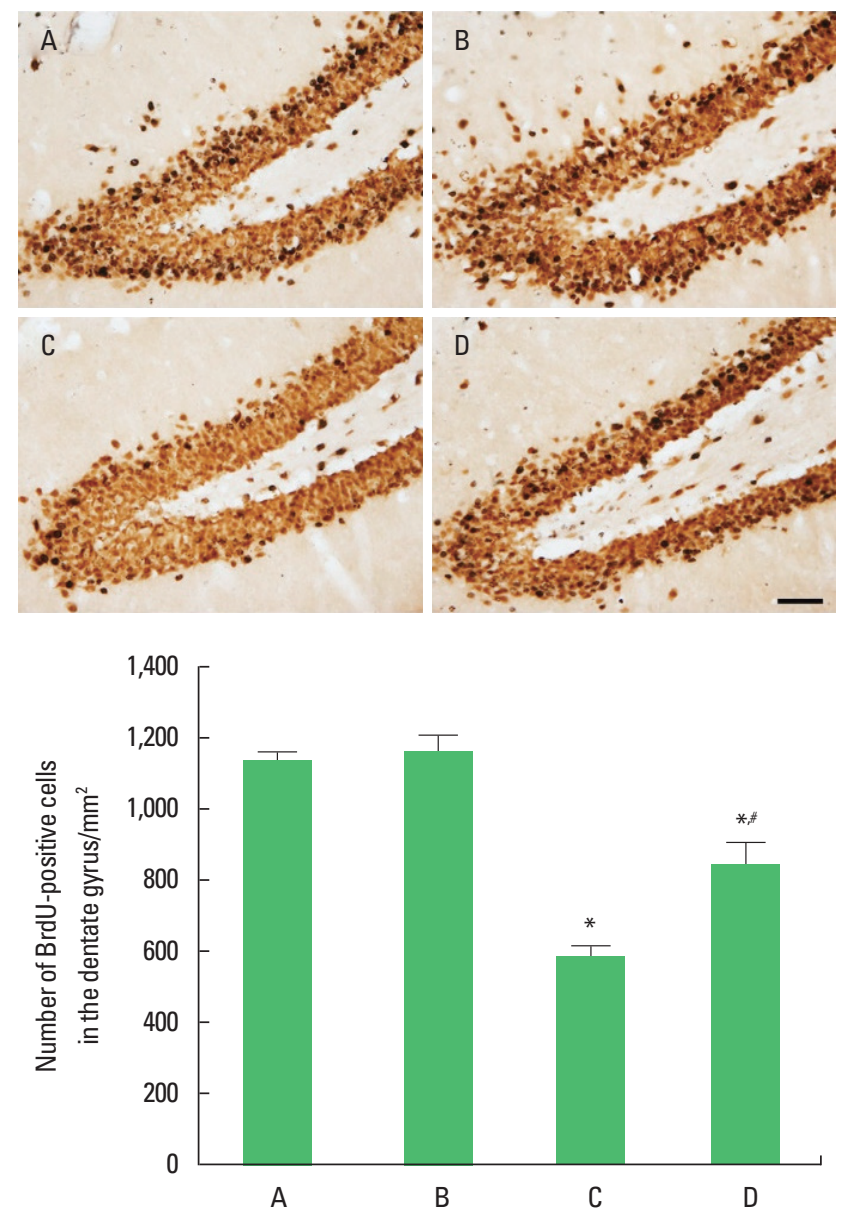

Fig. 3. Cell proliferation in the hippocampal dentate gyrus of rat pups. Upper panel: photomicrographs of 5-bromo-2'-deoxyuridine (BrdU)-positive cells. The scale bar represents $50 \mu \mathrm{m}$. Lower panel: number of BrdU-positive cells in each group. $A$, control group; $B$, treadmill exercise group; $C$, high-fat diet group; $D$, high-fat diet and treadmill exercise group. ${ }^{*} P<0.05$ compared with the control group. ${ }^{\sharp} P<0.05$ compared with the high-fat diet group. Data represents the mean \pm standard error of the mean.

with the control group $(P<0.05)$. Treadmill running was found to significantly increase the expression of BDNF and TrkB in the high-fat diet group $(P<0.05)$.

\section{DISCUSSION}

Ingestion of a high-fat diet during pregnancy enhances inflammatory cytokines, affecting the neurological development of offspring (Sullivan et al., 2014), and it has been reported that the offspring's behavior becomes abnormal (Sullivan et al., 2010). During pregnancy and lactation, the mother's intake of saturated transfat and high-fat diet decreases cognitive function (Bilbo and Tsang, 2010), and reduces neurotrophic factors associated with 


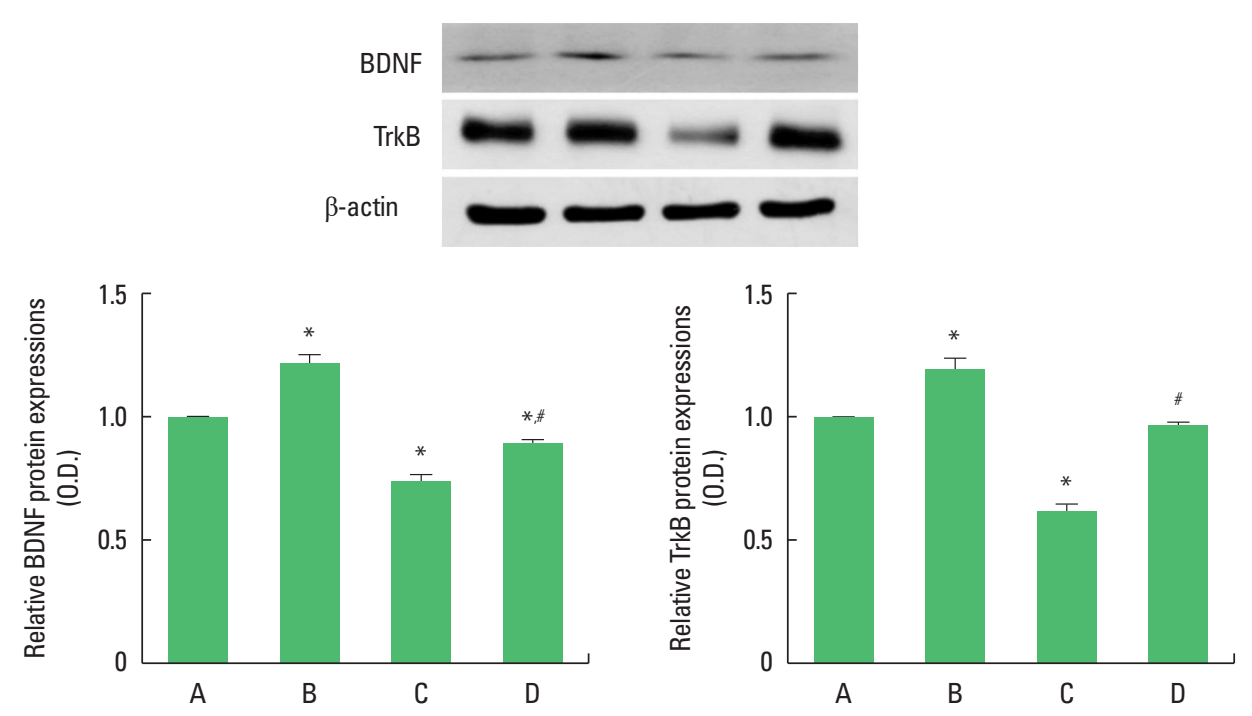

Fig. 4. Brain-derived neurotrophic factor (BDNF) and tyrosine kinase B (TrkB) expression in the hippocampus of rat pups. Upper panel: western blot analysis of BDNF and TrkB in the hippocampus. Left lower panel: optical density of BDNF expression in each group. Right lower panel: optical density of TrkB expression in each group. A, control group; $B$, treadmill exercise group; $C$, high-fat diet group; $D$, high-fat diet and treadmill exercise group. ${ }^{*} P<0.05$ compared with the control group. ${ }^{\#} P<0.05$ compared with the high-fat diet group. Data represents the mean \pm standard error of the mean.

cognitive function in the hippocampus of the brain (Tozuka et al., 2010). On the contrary, exercise is well known as a representative factor that improves cognitive function, and it has been reported that exercise enhances learning ability and memory function by increasing the production of neurons in the hippocampus (Trejo et al., 2001). Mother's exercise during pregnancy increases the offspring's ability to learn and memory (Akhavan et al., 2008; Kim et al., 2007) and increases neurogenesis in the hippocampus of pups born under stress (Kim et al., 2013). In the results of current study, short-term memory was reduced in the high-fat diet group compared with the control group. Treadmill running enhanced short-term memory in the high-fat diet rat pups.

The hippocampus belongs to the limbic system of the brain and is an important brain part involved in the formation of learning and memory in relation to neurogenesis. It is also the site of the greatest neuroplasticity (Snyder et al., 2005). During pregnancy or lactation, maternal nutrition and hormones exert critical effect in fetal growth and neural circuit formation in the brain (Férézou-Viala et al., 2007). Swimming exercise in maternal rats during pregnancy improved short-term memory and neuronal production in offspring (Lee et al., 2006), and treadmill exercise of rats during pregnancy enhanced the brain function in offspring (Kim et al., 2007). In the results of current study, the number of DCX-positive cells and BrdU-positive cells in the hippocampus was decreased in the high-fat diet group compared with the control group. Treadmill running increased the number of DCX-pos- itive cells and BrdU-positive cells in the high-fat diet rat pups.

BDNF is secreted from various brain regions and is an important representative substance for the growth and development of nerve cells and neuroplasticity. BDNF improves the survival of neurons by increasing resistance to nerve damage (Mizuno et al., 2000) and plays an important role in neuronal production in the hippocampus (Donovan et al., 2008). Ingestion of a high-fat diet reduced the production of neurons and expression of BDNF in the hippocampus (Stranahan et al., 2008), and long-term consumption of a high-fat diet impaired hippocampal function, which is responsible for learning and memory (Tozuka et al., 2010). BDNF regulates the development and differentiation of neurons by binding to the receptor TrkB, and BDNF is secreted by an activity-dependent manner (Bramham and Messaoudi, 2005). In the results of current study, the expression of BDNF and TrkB in the hippocampus was decreased in the high-fat diet group compared with the control group. Treadmill running enhanced BDNF and TrkB expression in the high-fat diet rat pups.

The current study revealed that neuronal cell generation and survival in the hippocampus of rat pups were increased through maternal treadmill exercise in the high-fat diet group. Treadmill exercise in the obese maternal rats increased BDNF and TrkB expression in offspring born to obese mothers, which has a positive effect on neuronal cell production and survival. 


\section{CONFLICT OF INTEREST}

No potential conflict of interest relevant to this article was reported.

\section{ACKNOWLEDGMENTS}

This work was supported by 2019 Sangmyung University Research Foundation of Korea.

\section{REFERENCES}

Akhavan MM, Emami-Abarghoie M, Safari M, Sadighi-Moghaddam B, Vafaei AA, Bandegi AR, Rashidy-Pour A. Serotonergic and noradrenergic lesions suppress the enhancing effect of maternal exercise during pregnancy on learning and memory in rat pups. Neuroscience 2008; 15:1173-1183.

Bilbo SD, Tsang V. Enduring consequences of maternal obesity for brain inflammation and behavior of offspring. FASEB J 2010;24:2104-2115.

Bramham CR, Messaoudi E. BDNF function in adult synaptic plasticity: the synaptic consolidation hypothesis. Prog Neurobiol 2005;76:99-125.

Chang GQ, Gaysinskaya V, Karatayev O, Leibowitz SF. Maternal high-fat diet and fetal programming: increased proliferation of hypothalamic peptide-producing neurons that increase risk for overeating and obesity. J Neurosci 2008;28:12107-12119.

Couillard-Despres S, Winkler J, Uyanik G, Aigner L. Molecular mechanisms of neuronal migration disorders, quo vadis? Curr Mol Med 2001;1:677-688.

Donovan MH, Yamaguchi M, Eisch AJ. Dynamic expression of TrkB receptor protein on proliferating and maturing cells in the adult mouse dentate gyrus. Hippocampus 2008;18:435-439.

Férézou-Viala J, Roy AF, Sérougne C, Gripois D, Parquet M, Bailleux V, Gertler A, Delplanque B, Djiane J, Riottot M, Taouis M. Long-term consequences of maternal high-fat feeding on hypothalamic leptin sensitivity and diet-induced obesity in the offspring. Am J Physiol Regul Integr Comp Physiol 2007;293:1056-1062.

Kim H, Lee SH, Kim SS, Yoo JH, Kim CJ. The influence of maternal treadmill running during pregnancy on short-term memory and hippocampal cell survival in rat pups. Int J Dev Neurosci 2007;25:243-249.

Kim TW, Shin MS, Park JK, Shin MA, Lee HH, Lee SJ. Treadmill exercise alleviates prenatal noise stress-induced impairment of spatial learning ability through enhancing hippocampal neurogenesis in rat pups. J Exerc Rehabil 2013;9:451-456.

Ko IG, Kim CJ, Kim H. Treadmill exercise improves memory by up-regu- lating dopamine and down-regulating D2 dopamine receptor in traumatic brain injury rats. J Exerc Rehabil 2019;15:504-511.

Lee HH, Kim H, Lee JW, Kim YS, Yang HY, Chang HK, Lee TH, Shin MC, Lee MH, Shin MS, Park S, Baek S, Kim CJ. Maternal swimming during pregnancy enhances short-term memory and neurogenesis in the hippocampus of rat pups. Brain Dev 2006;28:147-154.

Massa SM, Yang T, Xie Y, Shi J, Bilgen M, Joyce JN, Nehama D, Rajadas J, Longo FM. Small molecule BDNF mimetics activate TrkB signaling and prevent neuronal degeneration in rodents. J Clin Invest 2010;120: 1774-1785.

Mizuno M, Yamada K, Olariu A, Nawa H, Nabeshima T. Involvement of brain-derived neurotrophic factor in spatial memory formation and maintenance in a radial arm maze test in rats. J Neurosci 2000;20:71167121.

Park SS, Shin MS, Park HS, Kim TW, Kim CJ, Lim BV. Treadmill exercise ameliorates nicotine withdrawal-induced symptoms. J Exerc Rehabil 2019;15:383-391.

Seo JH, Kim TW, Kim CJ, Sung YH, Lee SJ. Treadmill exercise during pregnancy ameliorates post traumatic stress disorder induced anxiety like responses in maternal rats. Mol Med Rep 2013;7:389-395.

Shankar K, Harrell A, Liu X, Gilchrist JM, Ronis MJ, Badger TM. Maternal obesity at conception programs obesity in the offspring. Am J Physiol Regul Integr Comp Physiol 2008;294:528-538.

Snyder JS, Hong NS, McDonald RJ, Wojtowicz JM. A role for adult neurogenesis in spatial long-term memory. Neuroscience 2005;130:843-852.

Stranahan AM, Norman ED, Lee K, Cutler RG, Telljohann RS, Egan JM, Mattson MP. Diet-induced insulin resistance impairs hippocampal synaptic plasticity and cognition in middle-aged rats. Hippocampus 2008;18:1085-1088.

Sullivan EL, Grayson B, Takahashi D, Robertson N, Maier A, Bethea CL, Smith MS, Coleman K, Grove KL. Chronic consumption of a high-fat diet during pregnancy causes perturbations in the serotonergic system and increased anxiety-like behavior in nonhuman primate offspring. J Neurosci 2010;30:3826-3830.

Sullivan EL, Nousen EK, Chamlou KA. Maternal high fat diet consumption during the perinatal period programs offspring behavior. Physiol Behav 2014;123:236-242.

Tozuka Y, Kumon M, Wada E, Onodera M, Mochizuki H, Wada K. Maternal obesity impairs hippocampal BDNF production and spatial learning performance in young mouse offspring. Neurochem Int 2010; 57:235-247.

Trejo JL, Carro E, Torres-Aleman I. Circulating insulin-like growth factor I mediates exercise-induced increases in the number of new neurons in the adult hippocampus. J Neurosci 2001;21:1628-1634. 